\title{
ERRATUM
}

\section{Intrathyroidal Branchial Cleft-like Cyst with Heterotopic Salivary Gland-type Tissue}

Ji-Young Park, ${ }^{1}$ Gou Young Kim, ${ }^{2}$ and YeOn-Lim SuH ${ }^{1}$

${ }^{1}$ Department of Pathology, Samsung Medical Center, Sungkyunkwan University School of Medicine, Seoul 135-710, Korea

${ }^{2}$ Development of Pathology, Gachon Medical School Gil Medical Center, Incheon 405-760, Korea

(Pediatric and Developmental Pathology 7, 262-267, 2004, DOI: 10.1007/ s10024-003-3022-9)

The affiliation of Yeon-Lim Suh was published incorrectly, and it is corrected here. 